\title{
Adsorption-Photometric and Test-Determination of Fe(III) in Natural Water Using Silica Lyer-by-Lyer Modified with Polyhexamethylene Guanidine and Ferron
}

\author{
Svetlana L. Didukh-Shadrina, \\ Olga V. Buyko* and Vladimir N. Losev \\ Research Engineering Centre 'Kristall' \\ of Siberian Federal University \\ Krasnoiarsk, Russian Federation
}

Received 03.03.2020, received in revised form 04.03.2020, accepted 26.08.2020

\begin{abstract}
Silica based adsorbent sequentially modified with polyhexamethylene guanidine and 7-iodine-8-hydroxyquinoline-5-sulfonic acid (Ferron) was proposed for adsorption-photometric and test-determination of $\mathrm{Fe}(\mathrm{III})$ in natural water. During adsorption of $\mathrm{Fe}(\mathrm{III})$ from solutions with $\mathrm{pH}$ 2.0-3.5 adsorbent became green colored. Formation of green-colored surface complex of Fe(III) with Ferron with the maximum in diffuse reflectance spectrum at $600 \mathrm{~nm}$ was used for developing the procedure of its adsorption-photometric determination. Detection limit calculated by $3 \mathrm{~s}$-criterion was $0.03 \mu \mathrm{g} / 0.1 \mathrm{~g}$ of adsorbent ( $3 \mu \mathrm{g} / \mathrm{L}$ if the adsorption was carried out from $10 \mathrm{~mL}$ of solution); the analytical range was $10-220 \mu \mathrm{g} / \mathrm{L}$. Test-method was proposed for quick visual determination of $\mathrm{Fe}$ (III) in natural water in the variant of test-scale. Developed procedures were tested for determination of iron in natural river water of Krasnoyarskii kray and mineral water "Zagorie".
\end{abstract}

Keywords: adsorption-photometric determination, test-determination, iron(III), modified silicas, Ferron.

Citation: Didukh-Shadrina S.L., Buyko O.V., Losev V.N. Adsorption-photometric and test-determination of Fe(III) in natural water using silica lyer-by-lyer modified with polyhexamethylene guanidine and ferron, J. Sib. Fed. Univ. Chem., 2020, 13(3), 349-362. DOI: $10.17516 / 1998-2836-0188$

(C) Siberian Federal University. All rights reserved

This work is licensed under a Creative Commons Attribution-NonCommercial 4.0 International License (CC BY-NC 4.0).

* Corresponding author E-mail address: obuyko@sfu-kras.ru 


\title{
Сорбционно-фотометрическое и тест-определение Fe(III) \\ в природных водах с использованием кремнезема, послойно модифицированного
}

\section{полигексаметиленгуанидином и ферроном}

\author{
С.Л. Дидух-Шадрина, О.В. Буйко, В.Н. Лосев \\ Научно-исследовательский инженерный ичентр «Кристалл» \\ Сибирского федерального университета \\ Российская Федерачия, Красноярск
}

\begin{abstract}
Аннотация. Сорбент на основе кремнезема, последовательно модифицированный полигексаметиленгуанидином и 7-йод-8-оксихинолин-5-сульфокислотой (феррон), предложен для сорбционно-фотометрического и тест-определения $\mathrm{Fe}(\mathrm{III})$ в природных водах. В процессе сорбции $\mathrm{Fe}(\mathrm{III})$ из растворов с $\mathrm{pH}$ 2.0-3.5 происходит окрашивание сорбента в зеленый цвет. Образование окрашенного поверхностного комплекса $\mathrm{Fe}(\mathrm{III}) \mathrm{c}$ ферроном с максимумом в спектре диффузного отражения при 600 нм положено в основу разработки методики его сорбционнофотометрического определения. Предел обнаружения, рассчитанный по 3s-критерию, составляет 0.03 мкг/0.1 г сорбента (3 мкг/л, при сорбции из 10 мл раствора), диапазон определяемых содержаний составляет 10 - 220 мкг/л. Предложена тест-методика для визуального экспрессного определения Fe(III) в природных водах в варианте цветовых шкал. Разработанные методики опробованы при определении железа в природных водах рек Красноярского края и в минеральной воде «Загорье».
\end{abstract}

Ключевые слова: сорбционно-фотометрическое определение, тест-системы, железо(III), модифицированные кремнеземы, феррон.

Цитирование: Дидух-Шадрина С.Л. Сорбционно-фотометрическое и тест-определение $\mathrm{Fe}(\mathrm{III})$ в природных водах с использованием кремнезема, послойно модифицированного полигексаметиленгуанидином и ферроном $/$ С.Л. Дидух-Шадрина, О.В. Буйко, В.Н. Лосев // Журн. Сиб. федер. ун-та. Химия, 2020. 13(3). С. 349-362. DOI: $10.17516 / 1998-2836-0188$

\section{Введение}

Железо относится к биоактивным металлам и играет важную роль в жизни растений, животных и человека. Широко распространено в окружающей среде в степенях окисления +2 и +3 , причем в природных наземных водах содержание $\mathrm{Fe}(\mathrm{III})$ значительно превышает содержание $\mathrm{Fe}(\mathrm{II})$ [1]. Присутствие ионов железа в природных и бытовых водах обусловлено как природным, так и антропогенным происхождением, в результате использования в сельском и домашнем хозяйстве, промышленности, строительстве, медицине.

Повышенное содержание железа в питьевой воде не только ухудшает ее органолептические параметры, но и может негативно сказываться на состояние здоровья [2]. В настоящее время в России ПДК железа составляет 0.1 и 0.3 мг/л для рыбохозяйственных и культурно- 
бытовых вод соответственно, а согласно Всемирной организации здравоохранения (ВОЗ) и европейским нормативам для питьевой воды -0.3 и 0.2 мг/л [3, 4].

Уже традиционными для определения железа стали атомно-спектроскопические и массспектрометрические методы [5], которые отличаются низкими пределами обнаружения и хорошей воспроизводимостью результатов, но требуют квалифицированного персонала, дорогостоящего оборудования, их затруднительно использовать в полевых условиях, а также для них может понадобиться специальная пробоподготовка образцов.

В связи с постоянно возрастающим интересом общества к экологической обстановке актуальна разработка простых экспрессных недорогих методик с достаточно низкими пределами обнаружения $\mathrm{Fe}(\mathrm{III})$, доступных и для людей, не имеющих специального образования в области аналитической химии. К таким методам можно отнести сорбционноспектроскопические [6-9] и визуальные методы с использованием хемосенсоров или тестсистем [10-16].

Сочетание сорбционного концентрирования с последующим цветометрическим или молекулярно-спектроскопическим определением непосредственно в фазе сорбента позволяет получать пределы обнаружения железа, сопоставимые с атомно-спектроскопическими методами. В качестве сорбентов в таких случаях применяют модифицированные полиметакрилатные матрицы [7], пенополиуретаны [9, 15], органические матрицы $[8,10,14]$ и неорганические оксиды [6].

Тест-определение не требует специального оборудования, концентрация микрокомпонента определяется визуально сравнением интенсивности окраски или люминесценции с заранее разработанной тест-шкалой, при этом могут использоваться как твердые носители, так и жидкие хемосенсоры.

В данной работе в качестве сорбента для концентрирования и определения железа(III) использован кремнезем, последовательно модифицированный полигексаметиленгуанидином и 7-йод-8-оксихинолин-5-сульфокислотой (феррон). В качестве реагента предложен феррон, поскольку известно, что он образует интенсивно окрашенный в зеленый цвет комплекс с железом(III) [17-19].

\section{Материалы и методика}

\section{Реактивы}

Исходный раствор 100 мкг/мл Fе(III) готовили разбавлением ГСО (7835-2000 МСО 0294:2002 (1 г/дм³)) в $0.1 \mathrm{M} \mathrm{HCl}$.

Раствор полигексаметиленгуанидин гидрохлорида (ПГМГ) Института экологотехнологических проблем (Москва, Российская Федерация) с концентрацией $7.5 \%$ получили растворением препарата в деионизованной воде. Раствор феррона (7-йод-8-оксихинолин-5сульфокислоты натриевая соль) фирмы Sigma-Aldrich с концентрацией $0.1 \%$ готовили растворением препарата в деионизованной воде, растворы реагента с меньшей концентрацией - растворением исходного в деионизованной воде.

Силохром С-120 (размер частиц 0.1-0.2 мм, удельная поверхность $~ 120$ м²/г, средний диаметр пор 45 нм) фирмы «Люминофор» (Ставрополь, Россия) использован в качестве матрицы для получения сорбентов.

$$
-351-
$$


В работе использовали $\mathrm{HCl}, \mathrm{HNO}_{3}$ «ос.ч.», дополнительно очищенные перегонкой на установке distillacid ${ }^{\mathrm{TM}}$ BSB-939-IR (Berghof). Растворы кислот с меньшими концентрациями готовили разбавлением исходных растворов деионизованной водой.

Деионизованную воду с удельным сопротивлением $18.3 \mathrm{M} \Omega \mathrm{cm}^{-1}$ получали на установке E-pure D4642-33 (Barnstead International).

\section{Оборудование}

Спектры поглощения растворов феррона и комплекса $\mathrm{Fe}(\mathrm{III}) \mathrm{c}$ ферроном регистрировали на спектрофотометре Lambda 35 (Perkin-Elmer, США). Спектры диффузного отражения на поверхности сорбента регистрировали в диапазоне 380 - 720 нм на спектрофотометре «Пульсар» (НПО «Химавтоматика», Россия). Спектры диффузного отражения строили в координатах $F(R)-\lambda$, где $\lambda$ - длина волны, нм, а $F(R)$ - функция Гуревича-Кубелки-Мунка.

Концентрацию $\mathrm{Fe}(\mathrm{III})$ в растворах до и после сорбции определяли атомно-эмиссионным с индуктивно связанной плазмой методом на спектрометре iCAP 6500 (Thermo scientific, США) по градуировочным графикам. Построение градуировочных графиков и все расчеты проводили с помощью программного обеспечения прибора.

pH растворов до и после сорбции контролировали иономером Seven Multi (Mettler-Toledo, Швейцария).

Перистальтический насос MasterFlex L/S (Thermo Scientific, США) использован для прокачивания растворов при получении сорбента.

\section{Послойное модифицирование кремнезема}

полигексамителенгуанидином и ферроном

Для активирования поверхности кремнезем массой 5 г обрабатывали раствором $\mathrm{NaOH}$ при $\mathrm{pH}$ 8-9 в течение 2 ч. Затем промывали деионизованной водой до рН 7. Далее к сорбенту с помощью перистальтического насоса добавляли 100 мл раствора ПГМГ (7.5\%) со скоростью потока раствора 2 мл/мин. Полученный сорбент $\mathbf{S i O}-$-ПГМГ промывали деионизованной водой до исчезновения положительной реакции промывной воды на ПГМГ. Присутствие ПГМГ в промывной воде определяли визуально по синему окрашиванию с бромфеноловым синим.

К полученному сорбенту добавляли 100 мл раствора феррона с концентрацией $2.5 \cdot 10^{-5}$ $2.2 \cdot 10^{-3} \mathrm{M}$, перемешивали в течение 30 мин. После раствор декантировали, полученный сорбент $\mathrm{SiO}_{2}$-ПГМГ-феррон промывали водой и сушили на воздухе в течение суток. Этот сорбент не теряет своих сорбционных свойств в течение месяца.

Контроль за распределением феррона осуществляли по анализу водной фазы спектрофотометрическим методом по характеристической полосе реагента при 430 нм.

\section{Сорбиионное кониентрирование и сорбционно-фотометрическое}

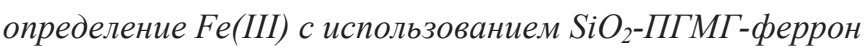

Сорбцию $\mathrm{Fe}(\mathrm{III})$ изучали в статическом режиме. Для этого в градуированные пробирки с притертой пробкой вносили 1 мл раствора, содержащего 0.05-25 мкг/мл $\mathrm{Fe}(\mathrm{III})$, добавляли $\mathrm{HNO}_{3}$ или $\mathrm{NaOH}$ для создания необходимого значения $\mathrm{pH}$, дистиллированную воду до объема 
10 мл. Вносили 0,1 г сорбента $\mathrm{SiO}_{2}$-ПГМГ-феррон, пробирки закрывали пробками и перемешивали в течение 1-30 мин.

Сорбент отделяли от раствора декантацией, помещали во фторопластовую кювету, измеряли коэффициент диффузного отражения. Контроль за распределением Fe(III) осуществляли атомно-эмиссионным с индуктивно связанной плазмой методом.

Отбор и пробоподготовка реальных образиов

и сорбиионно-люминесиентное определение Fe(III)

В качестве реальных объектов использовали образцы воды рек Базаиха, Кача, Чулым и минеральной воды «Загорье». Речную воду (200 мл) отбирали с глубины 0.5 м, фильтровали через целлюлозную мембрану (Millipore, 0.45 мкм), подкисляли азотной кислотой до $\mathrm{pH} 1$ и кипятили в течение 30 мин. После охлаждения отбирали аликвоту (10 мл), переносили в стеклянную пробирку с притертой крышкой, доводили $\mathrm{pH}$ до 3,0 добавлением $\mathrm{HCl}$, вносили 0.1 г сорбента $\mathrm{SiO}_{2}$-ПГМГ-феррон и перемешивали 10 мин. Раствор декантировали, сорбент переносили во фторопластовую кювету и измеряли коэффициент диффузного отражения при 600 нм.

\section{Pacчеть}

Степень извлечения $(R, \%) \mathrm{Fe}(\mathrm{III})$ сорбентом $\mathrm{SiO}_{2}$-ПГМГ-феррон рассчитывали по формуле

$$
R=\frac{C_{0}-C}{C_{0}} \times 100 \%
$$

где $C_{0}$ - исходная концентрация $\mathrm{Fe}(\mathrm{III})$, ммоль/л; $C$ - концентрация $\mathrm{Fe}(\mathrm{III})$, ммоль/л.

Сорбционную емкость $\left(q\right.$, ммоль/г) сорбента $\mathrm{SiO}_{2}$-ПГМГ по отношению к феррону рассчитывали по уравнению

$$
q=\frac{\left(C_{0}-C\right) \times V}{m}
$$

где $V$ - объем исследуемого раствора, л; $m$ - масса сорбента, г.

Предел обнаружения $\mathrm{Fe}(\mathrm{III})$ с использованием сорбента $\mathrm{SiO}_{2}$-ПГМГ-феррон рассчитывали по $3 s$-критерию.

\section{Результаты и обсуждение}

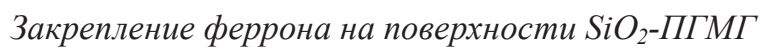

Непосредственное закрепление феррона на поверхности кремнезема невозможно из-за взаимного отталкивания отрицательно заряженных сульфогрупп реагента и депротонированных силанольных групп кремнезема. Предварительная обработка кремнезема водным раствором ПГМГ, в составе которого имеются положительно заряженные гуанидиновые группы, придает поверхности положительный заряд. Закрепление самого реагента осуществляется за счет электростатического взаимодействия между положительно заряженными гуанидиновыми группами $\mathrm{SiO}_{2}-$ ПГМГ и отрицательно заряженной сульфогруппой феррона (рис. 1).

Время установления сорбционного равновесия при извлечении феррона сорбентом $\mathrm{SiO}_{2}$ ПГМГ не превышает 10 мин.

$$
-353-
$$


<smiles></smiles>

Рис. 1. Схема сорбента $\mathrm{SiO}_{2}$-ПГМГ-феррон

Fig. 1. Scheme of the adsorbent $\mathrm{SiO}_{2}$-PHMG-Ferron

Феррон эффективно (97-99\%) закрепляется на поверхности $\mathrm{SiO}_{2}$-ПГМГ в диапазоне $\mathrm{pH}$ 2.0-7.0.

Сорбционная емкость $\mathrm{SiO}_{2}$-ПГМГ по феррону составляет 100 мкмоль/г. В процессе сорбции реагента сорбент окрашивается в желтый цвет $\left(\lambda_{\text {макс }}=430\right.$ нм), что при высоких поверхностных концентрациях реагента затрудняет спектрофотометрическое и визуальное определение низких концентраций $\mathrm{Fe}(\mathrm{III})$. Поэтому в работе использовали сорбент $\mathrm{SiO}_{2}$-ПГМГ-феррон с поверхностной концентрацией реагента 2.9 и 5.8 мкмоль на 0.1 г сорбента.

\section{Сорбиионное концентрирование}

и сорбционно-фотометрическое определение $\mathrm{Fe}(\mathrm{III})$ на $\mathrm{SiO}_{2}$-ПГМГ-феррон

B процессе сорбции $\mathrm{Fe}(\mathrm{III})$ на поверхности сорбента образуется комплексное соединение, окрашенное в зеленый цвет. Спектр диффузного отражения $\mathrm{Fe}(\mathrm{III})$ на поверхности $\mathrm{SiO}_{2}$-ПГМГферрон представляет собой широкую полосу с максимумами при 450 и 600 нм соответственно (рис. 2). Комплекс Fe(III) с ферроном в растворе при $\mathrm{pH}$ 2.0-5.0 имеет аналогичные спектроскопические характеристики (рис. 2), что свидетельствует о сохранении хромофорных свойств феррона на поверхности $\mathrm{SiO}_{2}$-ПГМГ.

Количественное извлечение (95-99\%) $\mathrm{Fe}(\mathrm{III})$ сорбентом $\mathrm{SiO}_{2}$-ПГМГ-феррон достигается в диапазоне $\mathrm{pH} 2.0-5.0$ и не зависит от поверхностной концентрации реагента. Максимальная интенсивность окраски поверхностного комплекса Fe(III) наблюдается при pH 2.0-3.5 (рис. 3). Аналогичный узкий диапазон рH (1.6-2.9) образования окрашенного комплекса Fe(III) c ферроном наблюдался при его экстракции хлороформом [19].

Время развития максимальной интенсивности окраски на поверхности $\mathrm{SiO}_{2}$-ПГМГ-феррон совпадает с временем установления сорбционного равновесия при извлечении $\mathrm{Fe}(\mathrm{III})$ и составляет 10 мин (рис. 4). 


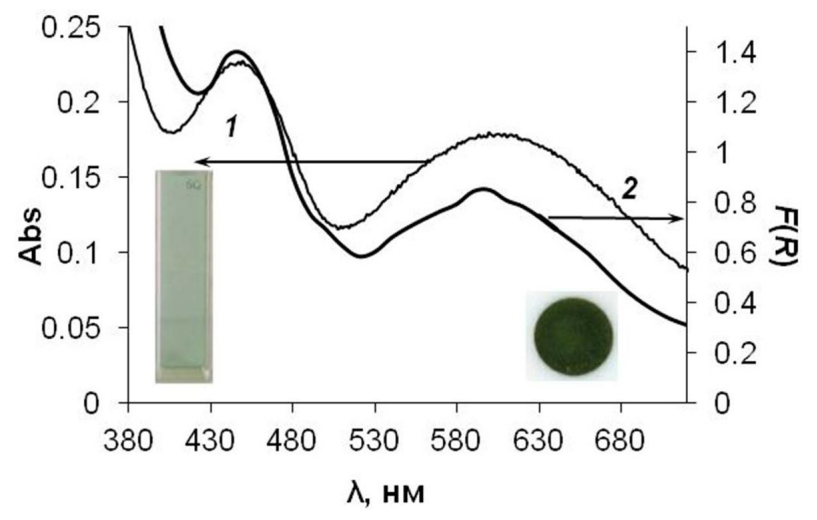

Рис. 2. Спектры поглощения (1) комплекса $\mathrm{Fe}(\mathrm{III})$ с ферроном в растворе и диффузного отражения (2) на поверхности $\mathrm{SiO}_{2}$-ПГМГ (0.1 г $\mathrm{SiO}_{2}$-ПГМГ-феррон, $C_{\mathrm{Fe}}=1.1$ мкг/мл, $V=10$ мл, pH 2.5, $t=10$ мин)

Fig. 2. UV-Vis (1) and diffuse reflectance (2) spectra of Fe(III) complex with ferron in solution and on the surface

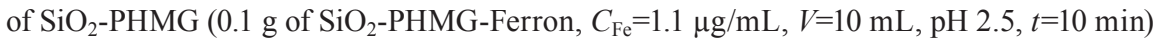

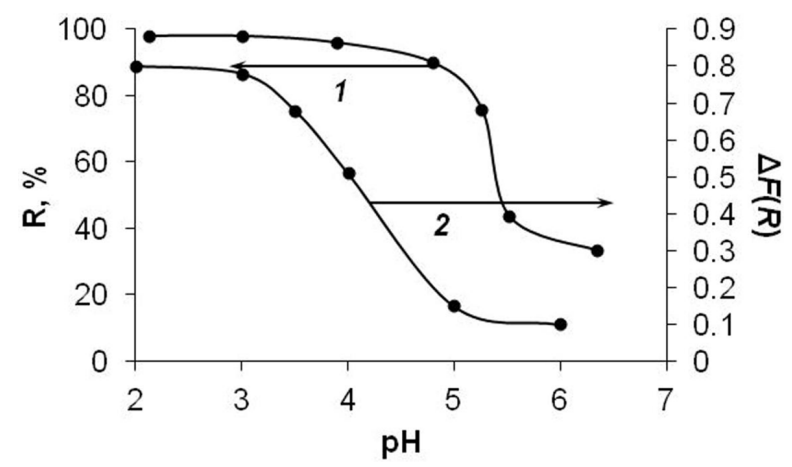

Рис. 3. Зависимость степени извлечения (1) и интенсивности окраски (2) $\mathrm{Fe}(\mathrm{III})$ на поверхности $\mathrm{SiO}_{2}$ ПГМГ-феррон от рН (0.1 г $\mathrm{SiO}_{2}$-ПГМГ-феррон, $C_{\mathrm{Fe}}=0.4$ мкг/мл, $V=10$ мл, $t=10$ мин)

Fig. 3. Dependence of extraction degree (1) and color intensity (2) of Fe(III) on the surface of $\mathrm{SiO}_{2}$-PHMG-Ferron vs. $\mathrm{pH}\left(0.1 \mathrm{~g}\right.$ of $\mathrm{SiO}_{2}$-PHMG-Ferron, $\left.C_{\mathrm{Fe}}=0.4 \mu \mathrm{g} / \mathrm{mL}, V=10 \mathrm{~mL}, t=10 \mathrm{~min}\right)$

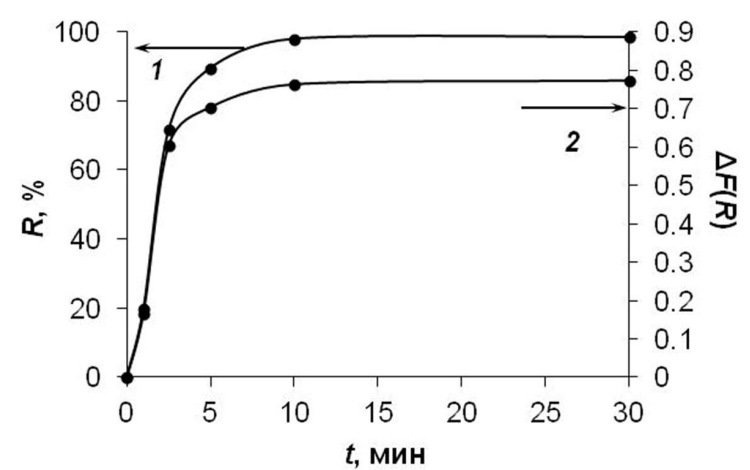

Рис. 4. Зависимость степени извлечения (1) и интенсивности окраски (2) $\mathrm{Fe}(\mathrm{III})$ на поверхности $\mathrm{SiO}_{2}$ ПГМГ-феррон от времени контакта фаз (0.1 г $\mathrm{SiO}_{2}$-ПГМГ-феррон, $C_{\mathrm{Fe}}=0.4$ мГ/л, $V=10$ мл, pH 2.5)

Fig. 4. Dependence of extraction degree (1) and color intensity (2) of Fe(III) on the surface of $\mathrm{SiO}_{2}$-PHMG-Ferron vs. phase contact time $\left(0.1 \mathrm{~g}\right.$ of $\mathrm{SiO}_{2}$-PHMG-Ferron, $\left.C_{\mathrm{Fe}}=0.4 \mu \mathrm{g} / \mathrm{mL}, V=10 \mathrm{~mL}, \mathrm{pH} 2.5\right)$ 


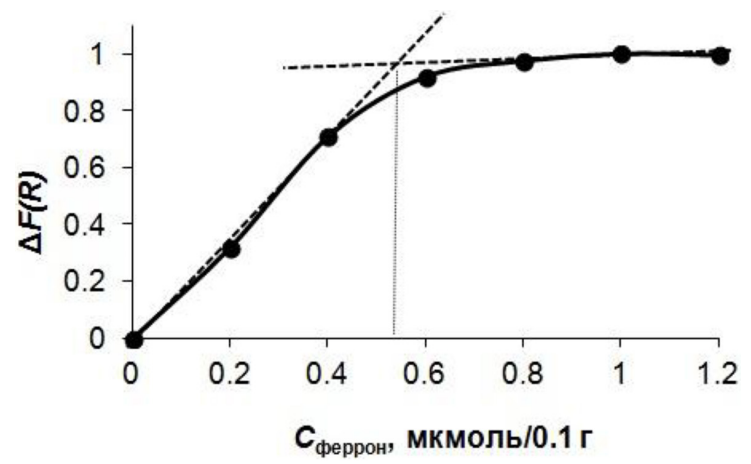

Рис. 5. Зависимость интенсивности окраски поверхностного комплекса $\mathrm{Fe}(\mathrm{III})$ от концентрации феррона на поверхности сорбента (0.1 г $\mathrm{SiO}_{2}$-ПГМГ-феррон, $C_{\mathrm{Fe}}=1.0$ мкг/мл, $V=10$ мл, pH 2.5, $t=10$ мин, $\lambda=600$ нм)

Fig. 5. Dependence of the surface complex of Fe(III) color intensity vs. Ferron concentration on the surface of adsorbent $\left(0.1 \mathrm{~g}\right.$ of $\mathrm{SiO}_{2}$-PHMG-Ferron, $\left.C_{\mathrm{Fe}}=1.0 \mu \mathrm{g} / \mathrm{mL}, V=10 \mathrm{~mL}, \mathrm{pH} 2.5, t=10 \mathrm{~min}, \lambda=600 \mathrm{~nm}\right)$

Сорбент имеет хорошие кинетические характеристики. Так, при увеличении объема раствора, из которого проводили сорбцию $\mathrm{Fe}(\mathrm{III})$, с 10 до 20 мл время установления сорбционного равновесия не превышает 10 мин. При использовании 10 мл раствора и 0.1 г сорбента коэффициент концентрирования равен 100. При увеличении объема раствора до 20 мл коэффициент концентрирования равен 200.

Из кривой насыщения (рис. 5) определена стехиометрия поверхностного комплекса $\mathrm{Fe}(\mathrm{III})$ :феррон, равная 1:3. Комплексы $\mathrm{Fe}(\mathrm{III})$ с ферроном аналогичного состава образуются в растворах [20], что подтверждает сохранение комплексообразующих свойств феррона при его закреплении на поверхности $\mathrm{SiO}_{2}$-ПГМГ.

Селективность определения Fe(III) в природной воде

с использованием $\mathrm{SiO}_{2}$-ПГМГ-феррон

Для установления селективности определения создавали бинарные системы, содержащие $\mathrm{Fe}(\mathrm{III})$ на фоне возрастающей концентрации сопутствующего элемента. Сорбционнофотометрическому определению $\mathrm{Fe}(\mathrm{III})$ с концентрацией 0.4 мг/л $\left(0,1\right.$ г $\mathrm{SiO}_{2}$-ПГМГ-феррон, pH 2.5) не мешают в кратных количествах $\mathrm{Na}(\mathrm{I}), \mathrm{K}(\mathrm{I}), \mathrm{Ca}(\mathrm{II})$ (1000), $\mathrm{Mg}(\mathrm{II}), \mathrm{Sr}(\mathrm{II})$ (500), $\mathrm{Cr}$ (III) (20), $\mathrm{Al}(\mathrm{III})$ (10), Ni(II), $\mathrm{Cd}(\mathrm{II}), \mathrm{Zn}(\mathrm{II})$ (5), $\mathrm{Cu}(\mathrm{II}), \mathrm{Mn}(\mathrm{II})$ (2.5) и солевой фон до 2 г/л по $\mathrm{Na}_{2} \mathrm{SO}_{4}, \mathrm{NaCl}$, $\mathrm{Na}_{2} \mathrm{CO}_{3}$.

\section{Сорбиионно-фотометрическое определение $\mathrm{Fe}(\mathrm{III})$}

с использованием $\mathrm{SiO}_{2}$-ПГМГ-феррон

Образование окрашенных комплексов $\mathrm{Fe}(\mathrm{III})$ на поверхности $\mathrm{SiO}_{2}$-ПГМГ-феррона и увеличение интенсивности окраски при 600 нм с увеличением концентрации металла на поверхности сорбента (рис. 6) положено в основу разработки методики сорбционно-фотометрического определения $\mathrm{Fe}(\mathrm{III})$.

Предел обнаружения $\mathrm{Fe}(\mathrm{III})$, рассчитанный по $3 s$-критерию, при использовании сорбента $\mathrm{SiO}_{2}$-ПГМГ-феррон с поверхностной концентрацией реагента 2.9 мкмоль/г составляет 0.03 мкг на 0.1 г сорбента, что при сорбции из 10 мл раствора соответствует 3 мкг/л $\mathrm{Fe}(\mathrm{III})$. Увеличение 


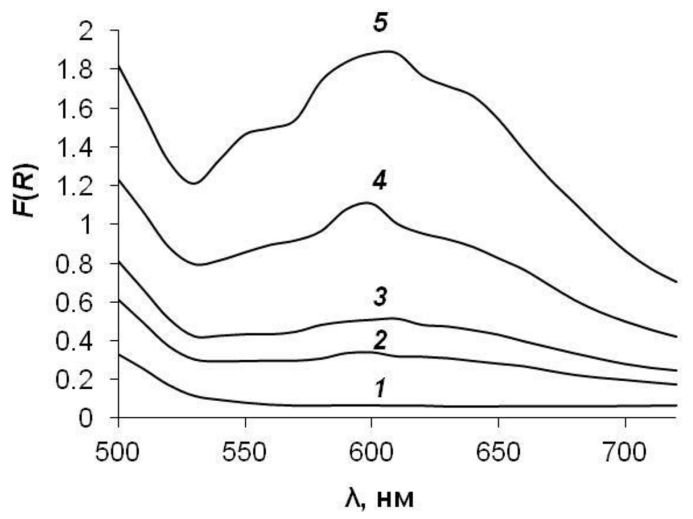

Рис. 6. Зависимость интенсивности окраски сорбента от концентрации $\mathrm{Fe}(\mathrm{III})$ на поверхности $\mathrm{SiO}_{2}$ ПГМГ-феррон (0.1 г $\mathrm{SiO}_{2}$-ПГМГ-феррон, $V=10$ мл, pH 2.5, $C_{\mathrm{Fe},}$ мкг/0,1 г сорбента: 0 (1), 1 (2), 2 (3), 6 (4), 11 $(5) ; n=3)$

Fig. 6. Dependence of color intensity of the adsorbent vs. Fe(III) concentration on the surface of $\mathrm{SiO}_{2}-\mathrm{PHMG}-$ Ferron (0.1 g of $\mathrm{SiO}_{2}$-PHMG-Ferron, $V=10 \mathrm{~mL}, \mathrm{pH}$ 2.5, $C_{\mathrm{Fe}}, \mu \mathrm{g} / 0,1 \mathrm{~g}$ of adsorbent: 0 (1), 1 (2), 2 (3), 6 (4), 11 (5); $n=3)$

Таблица 1. Метрологические характеристики сорбционно-фотометрического определения $\mathrm{Fe}(\mathrm{III})$ с использованием сорбента $\mathrm{SiO}_{2}$-ПГМГ-феррон (0.1 г сорбента, $V=10$ мл, $\left.\mathrm{pH} 2.5, n=3, P=0.95\right)$

Table 1. Analytical performance of adsorption-photometric determination of Fe(III) using $\mathrm{SiO}_{2}$-PHMG-Ferron adsorbent ( $0.1 \mathrm{~g}$ of adsorbent, $V=10$ мл, $\mathrm{pH} 2.5, n=3, P=0.95$ )

\begin{tabular}{cccccc}
\hline $\begin{array}{c}\text { Концентрация } \\
\text { феррона, мкмоль } / 0.1 \text { г }\end{array}$ & $\begin{array}{c}\text { Диапазон } \\
\text { определяемых } \\
\text { содержаний, мкг/0.1 г }\end{array}$ & $\begin{array}{c}\text { Предел обнаружения, } \\
\text { мкг/0.1 г }\end{array}$ & Уравнение & $R^{2}$ & $S_{r}$ \\
\hline 2.9 & $0.1-11$ & 0.03 & $\Delta F(R)=0.19 \cdot C_{\mathrm{Fe}}$ & 0.99 & 0.05 \\
& $10-110^{*}$ & $3^{*}$ & & & \\
& $0.1-22$ & 0.04 & $\Delta F(R)=0.19 \cdot C_{\mathrm{Fe}}$ & 0.99 & 0.05 \\
\hline
\end{tabular}

*Приведены значения в мкг/л при условии сорбции из 10 мл раствора на 0.1 г сорбента.

поверхностной концентрации реагента до 5.8 мкмоль/г приводит к увеличению предела обнаружения до 0.04 мкг на 0.1 г сорбента (табл. 1).

В табл. 2 сопоставлены пределы обнаружения $\mathrm{Fe}(\mathrm{III})$, полученные по разработанной методике, с опубликованными в литературе.

Как видно из данных табл. 2, предложенная методика сорбционно-фотометрического определения $\mathrm{Fe}(\mathrm{III})$ характеризуется пределом обнаружения ниже или сопоставимым с пределами обнаружения известных сорбционно-спектроскопических методик.

\section{Тест-определение $\mathrm{Fe}(\mathrm{III})$ с использованием $\mathrm{SiO}_{2}$-ПГМГ-феррон}

Изменение цвета сорбента $\mathrm{SiO}_{2}$-ПГМГ-феррон с желтого ( $\lambda=430$ нм) на зеленый ( $\lambda=600$ нм) при контакте с растворами, содержащими железо(III), и увеличение интенсивности его окраски с возрастанием концентрации металла на поверхности сорбента использовано при разработке методики тест-определения $\mathrm{Fe}(\mathrm{III})$ в природной и минеральной воде. 
Таблица 2. Сравнительные данные по определению $\mathrm{Fe}(\mathrm{III})$ в воде

Table 2. Comparative data of Fe(III) determination in water

\begin{tabular}{|c|c|c|c|}
\hline Метод & Сорбент & $\begin{array}{c}\text { Предел } \\
\text { обнаружения, мкг/л }\end{array}$ & Ссылка \\
\hline сорбционно-цветометрический & $\mathrm{NMCP}^{*}$ & 15 & {$[10]$} \\
\hline сорбционно-фотометрический & $\begin{array}{l}\text { акриловый полимер, } \\
\text { модифицированный койевой } \\
\text { кислотой }\end{array}$ & 2000 & {$[11]$} \\
\hline сорбционно-фотометрический & $\begin{array}{l}\text { акриловый полимер } \\
\text { с терпиридиновыми фрагментами }\end{array}$ & 7.3 & {$[12]$} \\
\hline $\begin{array}{l}\text { ПААС с предварительным } \\
\text { сорбционным концентрированием }\end{array}$ & $\begin{array}{l}\text { силикагель, модифицированный } \\
\text { 8-гидроксихинолином }\end{array}$ & 1.1 & {$[21]$} \\
\hline сорбционно-цветометрический & $\begin{array}{l}\text { пенополиуретан, } \\
\text { импрегнированный галловой } \\
\text { и сульфосалициловой кислотами }\end{array}$ & $\begin{array}{l}300 \\
500\end{array}$ & [9] \\
\hline $\begin{array}{l}\text { фотометрический } \\
\text { с предварительным сорбционным } \\
\text { концентрированием }\end{array}$ & $\begin{array}{l}\text { смола Dowex } 2 \mathrm{X} 4 \text {, } \\
\text { модифицированная ферроном }\end{array}$ & 10 & {$[22]$} \\
\hline сорбционно-фотометрический & 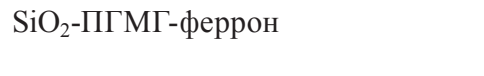 & 3.0 & $\begin{array}{l}\text { данная } \\
\text { работа }\end{array}$ \\
\hline
\end{tabular}

*Фильтровальная бумага, модифицированная N-допированным $\mathrm{MoO}_{3}$.

Поскольку сорбент $\mathrm{SiO}_{2}$-ПГМГ-феррон имеет собственную окраску, то на визуально минимально определяемую концентрацию $\mathrm{Fe}(\mathrm{III})$ оказывает влияние концентрация реагента на поверхности сорбента. На рис. 7 приведена фотография сорбентов, содержащих на поверхности 0.5 мкг $\mathrm{Fe}(\mathrm{III})$ и разные концентрации феррона.

Уменьшение концентрации феррона на поверхности сорбента увеличивает контрастность визуального определения $\mathrm{Fe}(\mathrm{III})$ и, как следствие, снижение визуально минимально определяемой концентрации. При разработке тест-шкалы использован сорбент $\mathrm{SiO}_{2}$-ПГМГферрон с поверхностной концентрацией реагента 2.9 мкмоль/0.1 г сорбента, визуально минимально определяемая концентрация $\mathrm{Fe}(\mathrm{III})$ составляет 50 мкг/л при сорбции из 10 мл воды.

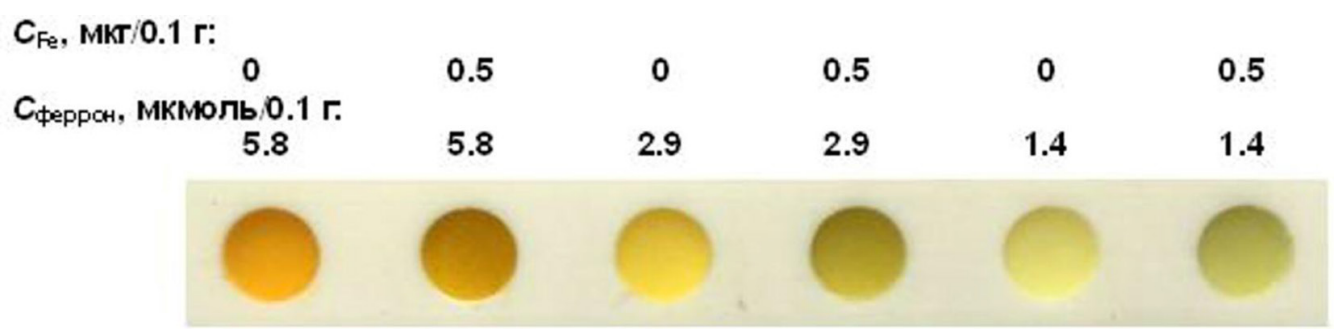

Рис. 7. Влияние концентрации феррона на поверхности $\mathrm{SiO}_{2}$-ПГМГ-феррон на визуальное определение 0.5 мкг $\mathrm{Fe}(\mathrm{III})$

Fig. 7. The effect of Ferron concentration on the surface of $\mathrm{SiO}_{2}$-PHMG-Ferron on the visual determination of $0.5 \mu \mathrm{g}$ of $\mathrm{Fe}$ (III) 


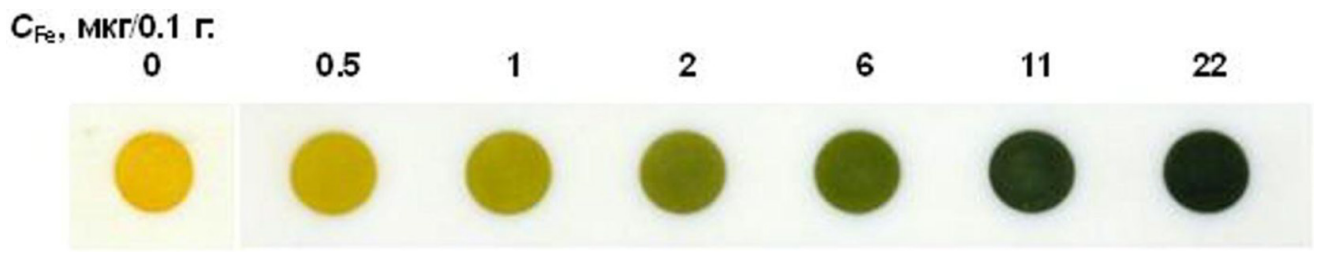

Рис. 8. Фотография цветовой шкалы для тест-определения $\mathrm{Fe}(\mathrm{III})$ в растворах с использованием сорбента и $\mathrm{SiO}_{2}-$ ПГМГ-феррон

Fig. 8. Photo of the color scale for the test-determination of $\mathrm{Fe}(\mathrm{III})$ in solutions using adsorbent $\mathrm{SiO}_{2}-\mathrm{PHMG}-$ Ferron

Изменение объема раствора, из которого осуществляется концентрирование $\mathrm{Fe}(\mathrm{III})$, с 5 до 20 мл не влияет на окраску сорбента и визуальное определение железа. Увеличение объема раствора до 30 мл приводит к снижению интенсивности окраски поверхностных комплексов железа и к заниженным значениям визуально определяемых концентраций.

Для создания цветовой шкалы 0.1 г сорбента $\mathrm{SiO}_{2}$-ПГМГ-феррон обрабатывают растворами, содержащими железо(III), объемом 10 мл и концентрацией 0-2.2 мг/л (pH 2.5, $t=10$ мин), сорбент отделяют от раствора декантацией и переносят в ячейки фторопластовой пластины в порядке возрастания концентрации металла на поверхности (рис. 8).

Определение Fe(III) в природной воде сорбционно-фотометрическим

и тест-методом с использованием $\mathrm{SiO}_{2}$-ПГМГ-феррон

Разработанные сорбционно-фотометрическая и тест-методики использованы при определении $\mathrm{Fe}(\mathrm{III})$ в природных водах рек Енисей и Кача и в бутилированной минеральной воде (табл. 3). Правильность полученных результатов подтверждена независимым атомноэмиссионным с ИСП методом.

Таблица 3. Результаты сорбционно-фотометрического и тест-определения Fe(III) в природных водах с использованием $\mathrm{SiO}_{2}$-ПГМГ-феррон (0,1 г $\mathrm{SiO}_{2}$-ПГМГ-феррон, $\mathrm{V}=10$ мл, pH 2.5, n=5, P=0.95)

Table 3. Results of adsorption-photometric and test-determination of $\mathrm{Fe}(\mathrm{III})$ in natural water using $\mathrm{SiO}_{2}$-PHMGFerron ( $0.1 \mathrm{~g}$ of $\mathrm{SiO}_{2}$-PHMG-Ferron, $V=10 \mathrm{~mL}, \mathrm{pH} 2.5, \mathrm{n}=5, \mathrm{P}=0.95$ )

\begin{tabular}{lccc}
\hline \multicolumn{1}{c}{ Образец } & \multicolumn{2}{c}{ Найдено, мкг/л } \\
& сорбционно-фотометрический & тест-метод & АЭС-ИСП \\
\hline р. Базаиха & $15.1 \pm 0.2$ & - & $15.2 \pm 0.1$ \\
р. Кача & $106 \pm 4$ & $100 \pm 25$ & $108 \pm 2$ \\
р. Чулым & $94 \pm 3$ & $100 \pm 25$ & $95 \pm 1$ \\
минеральная вода «Загорье» & $18 \pm 2^{*}$ & $20 \pm 5^{*}$ & $19 \pm 1^{*}$ \\
\hline
\end{tabular}

*Приведены значения в мг/л. 


\section{Заключение}

В работе описан простой способ получения сорбента на основе кремнезема, модифицированного полигексаметиленгуанидином и ферроном. Сорбционно-фотометрическое и тестопределение $\mathrm{Fe}(\mathrm{III})$ с использованием сорбента $\mathrm{SiO}_{2}$-ПГМГ-феррон позволяет проводить определение непосредственно в фазе сорбента. По достигаемому пределу обнаружения (3 мкг/л) разработанная сорбционно-фотометрическая методика превосходит или сопоставима с известными ранее методиками. Визуально минимально определяемая концентрация Fe(III) по разработанной тест-методике в варианте цветовых шкал составляет 50 мкг/л при сорбции из 10 мл воды. Методика может использоваться для определения Fe(III) в природных и минеральных водах.

\section{Благодарности / Acknowledgements}

Исследование выполнено при финансовой поддержке РФФИ и правительства Красноярского края в рамках научного проекта № 18-43-243004.

The reported research was funded by Russian Foundation for Basic Research and the government of Krasnoyarsk region of the Russian Federation, grant no. 18-43-243004.

\section{Список литературы / References}

1. Liu F.-J., Huang B.-Q., Li Sh.-X., Zheng F.-Y., Huang Xu-G. Effect of nitrate enrichment and diatoms on the bioavailability of Fe(III) oxyhydroxide colloids in seawater. Chemosphere 2016. Vol. 147, P. 105-113. doi:10.1016/j.chemosphere.2015.12.098

2. Ponka P., Tenenbein M., Eaton J.W. Iron. Handbook on the Toxicology of Metals, 3rd edition.: G.F. Nordberg, B.A. Fowler, M. Nordberg, L.T. Friberg. Amsterdam, Elsevier. 2007. P. 577-598. doi:10.1016/B978-012369413-3/50085-9

3. Ground Water and Drinking Water, EPA (2019) [Электронный ресурc]. http://www.epa.gov/ ground-water-and-drinking-water.

4. Drinking-water quality guidelines, World Health Organization (2019) [Электронный pecypc]. https://www.who.int/water_sanitation_health/water-quality/guidelines/en/

5. Pohl P., Sergiel I. Direct determination of the total concentrations of copper, iron and manganese and their fractionation forms in freshly ripened honeys by means of flame atomic absorption spectrometry. Microchimica Acta 2010. Vol. 168, P. 9-15.

6. Дидух С.Л., Мухина А.Н., Лосев В.Н. Сорбционно-фотометрическое и тест-определение общего содержания железа в природных водах с использованием сорбентов на основе оксида циркония, модифицированного полигексаметиленгуанидином, феррозином и ференом С. Аналитика и контроль 2014. T. 18(4), C. 430-437. [Didukh S.L., Mukhina A.N., Losev V.N. Sorptionphotometric and test-determination of total iron in natural waters using zirconium oxide based sorbents, modified with polyhexamethylene guanidine Ferrozine and Ferene S. Analytics and control 2014. Vol. 18(4), P. 430-437 (in Russ.)]

7. Gavrilenko N.A., Mokhova O.V. Sorption-spectrophotometric determination of iron(II, III) with the use of organic reagents immobilized in a polymethacrylate matrix. Journal of Analytical Chemistry 2008. Vol. 63(11), P. 1038-1043. doi: 10.1134/S106193480811004X

8. Shvoeva O.P., Dedkova V.P., Savvin S.B. Sorption-spectroscopy and test determination of uranium(VI) and iron(III) from a single sample on the solid phase of fiber materials filled with an 
AB-17 ion exchanger. Journal of Analytical Chemistry 2013. Vol. 68(10), P. 880-884. doi:10.1134/ S106193481308011X

9. Суханов П.Т., Калинкина С.П., Сердюкова К.С., Ильин А.Н. Сорбционноцветометрическое определение железа(III) в водах гальванического производства. Вестник ВГУИТ 2017. Т. 79(1), С. 215-221. [Sukhanov P.T., Kalinkina S.P., Serdiukova K.S., Ilin A.N. The sorption-chromaticity determination of Fe(III) in the waters of the galvanic production. Proceeding VSVET 2017. Vol. 79(1), P. 215-221 (in Russ.)]. doi:10.20914/2310-1202-2017-1-215-221

10. Lin F., Cai J., Li Yu., Yu Hu., Li Sh. Constituting fully integrated colorimetric analysis system for $\mathrm{Fe}(\mathrm{III})$ on multifunctional nitrogen-doped $\mathrm{MoO}_{3} /$ cellulose paper. Talanta 2018. Vol. 180, P. 352-357. doi:10.1016/j.talanta.2017.12.069

11. Vallejos S., Muñoz A., García F.C., Colleoni R., Biesuz R., Alberti G., García J.M. Colorimetric detection, quantification and extraction of $\mathrm{Fe}(\mathrm{III})$ in water by acrylic polymers with pendant Kojic acid motifs. Sensors and Actuators B: Chemical 2016. Vol. 233, P. 120-126. doi:10.1016/j.snb.2016.04.040

12. Trigo-López M., Muñoz A., Ibeas S., Serna F., García F.C., García J.M. Colorimetric detection and determination of $\mathrm{Fe}(\mathrm{III}), \mathrm{Co}(\mathrm{II}), \mathrm{Cu}(\mathrm{II})$ and $\mathrm{Sn}(\mathrm{II})$ in aqueous media by acrylic polymers with pendant terpyridine motifs. Sensors and Actuators B: Chemical 2016. Vol. 226, P. 118-126. doi:10.1016/j. snb.2015.11.116

13. Heo J., Manivannan R., Kim H., Kim M.J., Min K.S., Son Y. Developing an RGB - Arduino device for the multi-color recognition, detection and determination of $\mathrm{Fe}(\mathrm{III}), \mathrm{Co}$ (II), $\mathrm{Hg}$ (II) and $\mathrm{Sn}(\mathrm{II})$ in aqueous media by a terpyridine moiety. Sensors and Actuators B: Chemical 2019. Vol. 297, P. 126793. doi:10.1016/j.snb.2019.126723

14. Fan K., Wang Xi., Yu Sh., Han G., Xu D., Zhou L., Song J. A chitosan-based fluorescent hydrogel for selective detection of $\mathrm{Fe}^{2+}$ ions in gel-to-sol mode and turn-off fluorescence mode. Polymer Chemistry 2019. Vol. 10, P. 5037-5043. doi:10.1039/C9PY01179J

15. Ivkova T.I. The indicator system based on bathophenanthroline for rapid determination of Fe(II, III) in water. Journal of Water Chemistry and Technology 2008. Vol. 30(6), P. 368-374. doi:10.3103/S1063455X08060064

16. Tsay H.-T., Bhorge Y.R., Pape A.J. Janaki S.N., Yen Y.-P. A Selective Colorimetric and Fluorescent Chemodosimeter for Fe(III) Ion Based on Hydrolysis of Schiff Base. Journal of Chinese Chemical Society 2015. Vol. 62(4), P. 316-320. doi:10.1002/jccs.201400394

17. Swank H.W., Mellon M.G. Determination of iron: with 7-iodo-8-hydroxyquinoline-5-sulfonic acid. Industrial and Engineering Chemistry 1937. Vol. 9(9), P. 406-409. doi:10.1021/ac50113a002

18. Storm A.R., Langmyhr F.J. Complex formation of ferric iron with 7-iodo-8-hydroxy-quinoline5-sulfonic acid (Ferron). Acta Chemica Scandinavica 1961. Vol. 15, P. 1765-1771. doi:10.3891/acta. chem.scand.15-1765

19. Przeszlakowski S., Habrat E. Extraction of Iron(III) from Aqueous Solution with Mixtures of Aliquat 336 and Ferron in Chloroform. Analyst 1982. Vol. 107, P. 1320-1329. doi:10.1039/ AN9820701320

20. Zaky M., Hanna W.G., Nour E.M., Killa H.M. Complexation of Ferron with some Metal Ions of Biological Interest. Analytical Letters 1985. Vol. 18, P. 803-814. doi:10.1080/00032718508066178

21. Goswami A., Singh A.K., Venkataramani B. 8-Hydroxyquinoline anchored to silica gel via new moderate size linker: synthesis and applications as a metal ion collector for their flame atomic 
absorption spectrometric determination. Talanta 2003. Vol. 60, P. 1141-1154. doi:10.1016/S00399140(03)00218-2

22. Moldovan Z., Neagu E.-A. Spectrophotometric determination of trace iron(III) in natural water after its preconcentration with chelating resin. Journal of Serbian Chemical Society 2002. Vol. 67(10), P. 669-676. doi:10.2298/JSC0210669M 